\section{Time for an individualized approach to first-line management of follicular lymphoma}

\section{Guillaume Cartron ${ }^{1}$ and Judith Trotman ${ }^{2}$}

${ }^{1}$ Department of Hematology, University Hospital, Montpellier, France and ${ }^{2}$ Department of Hematology, Concord Hospital, Sydney, Australia

\section{ABSTRACT}

F ollicular lymphoma is a heterogeneous B-cell lymphoma both in presentation and at progression. For most patients it is a chronic, relapsing indolent disease with overall survival expectations now potentially beyond 20 years. However, in a significant minority $(\sim 20 \%)$ who experience early progression or histological transformation after treatment, the disease no longer has an indolent behavior. This review looks at the development of prognostic indices, staging and therapies for follicular lymphoma, identifying where the data can, and cannot, guide the multidisciplinary team to determine an individualized approach to first-line therapy. A nuanced patient- and disease-specific approach is necessary to maximize disease response and survival while minimizing therapeutic toxicity.

\section{Introduction}

Follicular lymphoma (FL) is an indolent lymphoid neoplasm derived from germinal center B cells. Rapid therapeutic initiation is rarely required. With the oftenadvanced age of patients at diagnosis and the indolent nature of the disease, some patients will never need treatment. Almost half of patients with asymptomatic disease undergoing an accepted "watch and wait strategy" will not have commenced treatment 31 months after diagnosis. ${ }^{1}$ For symptomatic patients, the combination of a monoclonal antibody against CD20 (rituximab, obinutuzumab) with chemotherapy results in $80 \%$ survival at 10 years. ${ }^{2}$ Many of these patients will not experience disease relapse or die from their disease, even though FL remains the most common cause of death. ${ }^{3}$ The challenge for hematologists is to identify patients who need treatment and to define the most appropriate treatment for them considering their age, comorbidities and likelihood of subsequent relapse, to optimize both longevity and quality of life. While most patients are destined to have a prolonged survival, described as a "functional cure", approximately $20 \%$ of patients will progress within the first 2 years of initiating treatment. These early failures, which frequently correspond with histological transformation into diffuse large B-cell lymphoma, constitute the true evolutionary turning point of this disease, accounting for more than $50 \%$ of patinets' deaths in the first decade. ${ }^{3}$ Faced with difficulty in predicting an individual patient's outcome before treatment initiation, it is important to implement a dynamic strategy that ensures the early identification of those patients who experience histological transformation in order to offer them innovative therapeutic strategies. The nuanced management of FL is now well charted. With our comprehensive knowledge of the disease, its evolution and patient-related factors, we are now able to discuss different therapeutic approaches with our patients. The recent arrival of severe acute respiratory syndrome coronavirus-2 (SARS-CoV-2) challenges certain therapeutic choices. Here again, our evolving knowledge and a balanced and honest discussion with the patient should enable informed decision-making adapted to the patient's life priorities.

\section{Epidemiology and environmental factors}

FL is the most frequent indolent lymphoma, representing around $20 \%$ of all adult lymphomas in Europe, $30 \%$ in the USA and 10\% in Asia and developing
Haematologica 2022

Volume 107(1):7-18

\section{Correspondence:}

GUILLAUME CARTRON

g-cartron@chu-montpellier.fr

Received: June 23, 2021.

Accepted: October 1, 2021.

https://doi.org/10.3324/haematol.2021.278766

(C)2022 Ferrata Storti Foundation

Material published in Haematologica is covered by copyright. All rights are reserved to the Ferrata Storti Foundation. Use of published material is allowed under the following terms and conditions:

https://creativecommons.org/licenses/by-nc/4.0/legalcode. Copies of published material are allowed for personal or internal use. Sharing published material for non-commercial purposes is subject to the following conditions:

https://creativecommons.org/licenses/by-nc/4.0/legalcode, sect. 3. Reproducing and sharing published material for commercial purposes is not allowed without permission in writing from the publisher. 
countries The median age at diagnosis is 63 years and the male/female ratio is around $1.2{ }^{4}$ The incidence is 2.7 per 100,000 people, such that there are approximately 13,200 new cases of FL in the USA each year. Most patients $(54.8 \%)$ are aged $55-75$ years whereas patients younger than 40 and older than 85 represent a minority (2.4 and $4.8 \%$, respectively). Epidemiological data from outside the USA remain sparse, but a French report charted a similar incidence and median age. ${ }^{5}$

A family history of non-Hodgkin lymphoma (odds ratio [OR]: 1.99), high body mass index in young adulthood (OR: 1.15) and Sjögren syndrome in young women (OR: 3.37) increase the risk of FL. ${ }^{6}$ Among environmental factors, smoking (especially heavy smoking in women, OR: 1.41) also correlated with FL.7 Prolonged agriculture exposure to pesticides increases the risk of $\mathrm{FL}^{8}$ (OR: 2.95.0 according to the type of pesticide), a situation also associated with both circulating $B$ cells harboring the initiating $t(14 ; 18)$ and genetic alterations linked to malignant progression. $^{9}$

\section{Clinical features and diagnostic approach}

Most patients with FL present with asymptomatic disease and the diagnosis is often suspected incidentally during a clinical or radiological examination performed for symptoms unrelated to FL. When symptomatic, the clinical presentation is dominated by lymphadenopathy or less frequently disease-related complications, such as abdominal pain (mesenteric lymphadenopathy) or respiratory signs (cough, shortness of breath, pleural effusion) in the event of thoracic lymphadenopathy. The general "B" symptoms (recurrent fever $>38^{\circ} \mathrm{C}$, drenching sweats, weight loss $>10 \%$ ) associated with lymphoma only occur in about $20 \%$ of patients and should raise the suspicion of histological transformation.

Laboratory investigations look for the existence of cytopenia, particularly anemia. While bone marrow infiltration is the most common cause, the possibility of autoimmune cytopenia should not be underestimated. The presence of circulating lymphoma cells, found in fewer than $10 \%$ of cases, is associated with a shorter progression-free survival (PFS). ${ }^{10}$ The lactate dehydrogenase and $\beta_{2}$-microglobulin levels are important prognostic markers, and serum electrophoresis should be systematically requested in the search for an associated monoclonal protein and viral serology (hepatitis B and C, human immunodeficiency virus) should be determined before any treatment using anti-CD20 monoclonal antibody.

The diagnosis of FL is made on a lymph node or, in the uncommon extranodal FL, organ biopsy. Initial diagnosis by fine needle aspiration or needle biopsy is not recommended due to the importance of architectural examination in diagnosis and the risk of missing histological transformation. Multiple core needle biopsies, guided by ultrasonography or a computerized tomography (CT) scan, using a 14- or 16-gauge needle, may be informative but should be reserved in the context of disease to abdominal or extranodal sites. If it remains debated, when clinical findings suggest a possible hidden histological transformation, a ${ }^{18}$ F-fluorodeoxyglucose (FDG) positron emission tomography, coupled with low-dose CT (PET) may assist in identifying sites where a repeat biopsy could identify such. There are however no prospective data to support re-biopsy on the basis of baseline quantitative PET metrics alone. Indeed, a large body of data from the GALLIUM study suggests no association of maximum standardized glucose uptake value (SUVmax) with either histological transformation ${ }^{11}$ or PFS. ${ }^{12}$ There is marked heterogeneity in FDG uptake within patients which reflects metabolic activity of the malignant $B$ cells as well as that of the microenvironment. ${ }^{13}$ Likewise, the isolated analysis of bone marrow or blood infiltration is not appropriate for the diagnosis of FL.

\section{Histopathology}

FL is an entity well-defined in the World Health Organization (WHO) classification. ${ }^{14}$ The macroscopic appearance of lymph nodes involved by FL is usually vaguely nodular with a predominantly follicular organization microscopically. Thus, tumors frequently maintain a normal germinal center architecture forming enlarged lymphoid follicles with centroblasts and centrocytes randomly distributed with a loss of the polarization usually seen in reactive lymph nodes. On immunochemistry, FL cells typically express a germinal center pattern (CD10, BCL6), B-markers (CD19, CD20, SIg) and BCL2. Although absent in 10 to $15 \%$ of cases, BCL2 overexpression, secondary to $t(14 ; 18)$, is the hallmark of FL and can be useful in distinguishing FL from reactive follicles. It is important to note that $\mathrm{t}(14 ; 18)$ ( $\mathrm{q} 32 ; \mathrm{q} 21)$, placing BCL2 under the transcriptional regulation of $I G H$ regulatory regions leading to the overexpression of this anti-apoptotic protein, is a lymphoma-initiating event. In cases lacking BCL2 expression, the usual BCL6 and CD10 expression helps to distinguish FL from other lymphoproliferative diseases. Present at low level in $70 \%$ of healthy individuals, lymphoid cells harboring $t(14 ; 18)$ require additional genetic events for the development of FL.

A grading system based on counting the absolute number of centroblasts in ten neoplastic follicles per high power field is used to characterize FL into grades 1 and 2 (grade 1: 0-5 centroblasts per high power field, grade 2: 615) which represent the majority of cases (80\%). Grade 3 FL has $>15$ centroblasts per high power field and is further subdivided into FL 3A (centrocytes still present) and FL 3B (composed exclusively of centroblasts). FL grade $3 B$ is now managed as an aggressive lymphoma similar to diffuse large B-cell lymphoma. Considering the therapeutic impact of distinguishing between FL3A/3B, an expert review of histological classification may be useful. Rare entities including testicular FL, duodenal-type FL, pediatric FL and in situ follicular neoplasia are also characterized in the WHO classification. These entities are not discussed in this review.

\section{Staging}

At diagnosis, staging is mandatory to determine the distribution and burden of the lymphoma. Staging is an important factor in guiding treatment decisions - influencing both choice and timing of therapy- and re-staging is important to assess disease response after treatment and at times of progression requiring treatment. The current staging procedures include physical examination, blood investigations (full blood count, renal function and liver function 
Table 1. Revised staging system for primary lymphoma (adapted from Cheson et al. ${ }^{76}$ ).

\begin{tabular}{lll}
$\begin{array}{ll}\text { Limited } \\
\text { I }\end{array}$ & $\begin{array}{l}\text { One or several nodes of one group } \\
\text { Two or more nodal groups on the same side } \\
\text { of the diaphragm }\end{array}$ & $\begin{array}{l}\text { Single extranodal } \\
\text { Stage I or II with contiguous and limited extranodal involvement }\end{array}$ \\
$\begin{array}{ll}\text { I or II bulky* } \\
\text { Idvanced }\end{array}$ & Not II as above with bulky disease \\
III & $\begin{array}{l}\text { Nodes on both sides of the diaphragm or nodes } \\
\text { above the diaphragm with spleen involvement }\end{array}$ & Not applicable \\
IV & Additional non-contiguous or extended extranodal involvement & Not applicable \\
\hline
\end{tabular}

*'Bulky' disease has been defined as any nodal mass of $10 \mathrm{~cm}$ or greater than one third of the transthoracic diameter at any level of thoracic vertebrae. Tonsils, Waldeyer ring and spleen are considered nodal sites.

Table 2. Criteria for initiation of treatment in patients with follicular lymphoma.

\begin{tabular}{|c|c|}
\hline GELF criteria ${ }^{27}$ & BNLI criteria ${ }^{28}$ \\
\hline Presence of at least one of the following criteria: & Presence of at least one of the following criteria: \\
\hline Any B symptom & Pruritus or B symptom(s) \\
\hline Involvement of $\geq 3$ nodal sites, each with a diameter $\geq 3 \mathrm{~cm}$ & Rapid generalized disease progression in the preceding 3 months \\
\hline Tumor mass $\geq 7 \mathrm{~cm}$ & Life-endangering organ involvement \\
\hline Symptomatic splenomegaly & Significant bone marrow infiltration \\
\hline Pleural effusion or ascites & Localized bone lesions detected on X-ray or isotope scan \\
\hline Organ compression & Renal infiltration \\
\hline Serum LDH or $\beta_{2}$ M above upper limit of normal & 'Macroscopic' as opposed to 'microscopic' liver involvement \\
\hline
\end{tabular}

tests, lactate dehydrogenase and $\beta_{2}$-microglobulin), CTscan of the neck, thorax, abdomen and pelvis and PET.

PET is now the recommended gold-standard imaging investigation at initial staging. FL has a universally, albeit not uniformly, glucose-avid histology, with FDG uptake in $98 \%$ of patients. ${ }^{15}$ PET is more sensitive than standard contrast-enhanced CT at detecting extranodal disease. ${ }^{16}$ The PET report should indicate nodal and extranodal ${ }^{18} \mathrm{~F}-$ FDG uptake due to lymphoma as well as the lesion with the highest standardized glucose uptake value (SUVmax). The extent of the disease is then classified according to the Lugano classification (Table 1). PET scanning resulted in up-staging of as many as $62 \%$ of localized (I/II) cases into advanced stage (III/IV) cases. ${ }^{17}$ The utility of PET has been demonstrated by identifying disseminated disease ultimately associated with a poorer outcome if treated as localized on the basis of CT-based staging. ${ }^{17}$ The prognostic value of total metabolic tumor volume at diagnosis in FL remains debated ${ }^{12,18}$ and the predictive value of automated software solutions for measuring total metabolic tumor volume ${ }^{19}$ needs to be validated in prospective studies before being utilized in a standard manner in clinical practice. The prognostic value of end-of-induction PET response after first-line immunochemotherapy for $\mathrm{FL}^{20,21,22}$ is now clear and provides a platform for PET-adapted therapies in current clinical trials..$^{23,24}$

While focal bone marrow involvement can be identified on PET, it is generally less sensitive than bone marrow biopsy because it is often diffuse and low volume. Thus, when identification of bone marrow involvement is necessary (cytopenia, clinical trial, confirmation of localized disease), bone marrow biopsy (including both aspirate and trephine) is required to complete marrow assessment when the PET is negative. Identification of marrow involvement does not change treatment in dis- seminated disease and its prognostic value is debated, with prognostic merit identified in the PRIMA study ${ }^{25}$ but not in the GALLIUM study. ${ }^{26}$

\section{Treatment-initiation criteria}

Tumor burden is usually considered an important prognostic factor in FL. This observation and the commonly indolent nature of FL led the Groupe d'Etude des Lymphomes Folliculaires (GELF) ${ }^{27}$ and the British National Lymphoma Investigation (BNLI) ${ }^{28}$ to propose criteria for initiation of treatment (Table 2). These are mainly clinical criteria, empirically defined in the 1990s. They are still used to guide initial management in both clinical practice and trials and have shown consistent prognostic value. ${ }^{29}$ Notwithstanding the value of such criteria, in the patient with a slowly waxing and waning disease, it may not be appropriate to commence treatment in routine clinical practice just because they have a mildly elevated lactate dehydrogenase or an asymptomatic abdominal mass that has taken years to reach $7 \mathrm{~cm}$ in longest dimension. Conversely, some young patients who do not strictly meet GELF/BNLI criteria may warrant initiation of therapy for rapidly progressing disease.

\section{Prognostic indices}

In 2004, the Follicular Lymphoma International Prognostic Index (FLIPI) was the first prognostic index dedicated to $\mathrm{FL}^{30}$ (Table 3). FLIPI separates three groups of patients (approximately one third each) with significant differences in overall survival (OS) (Table 4). Although originally designed in the pre-rituximab era, FLIPI has 
Table 3. Adverse prognostic factors according to FLIPI, FLIPI-2 and PRIMA-PI.

\begin{tabular}{|c|c|c|}
\hline$F L|P|^{30}$ & FLIPI-21 & PRIIMA-P| ${ }^{20}$ \\
\hline Age $>60$ years & Age $>60$ years & \\
\hline Ann Arbor stage III/IV & Marrow involvement & Marrow involvement \\
\hline LDH > normal & $\beta_{2}$-microglobulin $>$ normal & $\beta_{2}$-microglobulin $>3 \mathrm{mg} / \mathrm{L}$ \\
\hline Hemoglobin < $120 \mathrm{~g} / \mathrm{L}$ & Hemoglobin < $120 \mathrm{~g} / \mathrm{L}$ & \\
\hline Nodal sites involved $>4$ & Tumor mass $>6 \mathrm{~cm}$ & \\
\hline
\end{tabular}

FLIPI: Follicular Lymphoma International: Prognostic Index; PRIMA-PI; PRIMA Prognostic Index; LDH: lactate dehydrogenase.

been validated in studies using immunochemotherapy and is now widely used in daily practice and clinical trials. FLIPI-2 was developed in a population of rituximab-treated patients for whom $\beta_{2}$-microglobulin was available ${ }^{31}$ and is also predictive of PFS and OS. The PRIMA prognostic index (PRIMA-PI) was developed from a retrospective analysis of a population of patients receiving immunochemotherapy followed by rituximab maintenance ${ }^{25}$ in a large randomized clinical trial. This prognostic index is based on $\beta_{2}$-microglobulin and marrow infiltration, two easily available parameters, albeit with the requirement of an invasive bone marrow biopsy. Recently, with the description of recurrent gene mutations in FL, some groups have designed new prognostic indices, including both clinical factors and mutational status. ${ }^{32,33}$ The use of these indices is uncommon in routine practice due to the limited availability of mutational analysis, and they have only been validated as prognostic in the FL population treated with R-CHOP (rituximab plus cyclophosphamide, doxorubicin, vincristine, prednisone).

\section{Natural course of the disease}

FL is still considered as an indolent but usually incurable disease, although the age- and gender-matched survival in patients who have remained event-free for 2 years after initial treatment challenges our concepts of cure. ${ }^{34} \mathrm{FL}$ is characterized by a heterogeneous presentation and outcome reflecting its biological heterogeneity. Patients with advanced stage indolent disease are manageable with a "wait and watch" strategy for many years whereas others may experience short OS related to transformation into diffuse large B-cell lymphoma. In the former population, representing one third of patients, an indolent presentation does not require therapeutic intervention. ${ }^{35}$ For such asymptomatic patients, $50 \%$ and $20 \%$ will not require treatment at 3 and 10 years, respectively, after diagnosis and their estimated OS is probably higher than the median $80 \%$ charted at 10 years after rituximab chemotherapy. ${ }^{1,2,28}$ The pattern of clinical evolution seems to be the same for FL grades 1,2 and $3 \mathrm{~A}$ whereas, grade $3 \mathrm{~B} F \mathrm{~L}$ is now recognized to be genetically closer to diffuse large B-cell lymphoma with a more aggressive course that requires anthracycline-containing immunochemotherapy at diagnosis. ${ }^{36}$ For patients requiring treatment, the use of combined immunochemotherapy results in a 10-year OS of around $80 \%{ }^{2}$ However, the lack of a plateau in the PFS curve suggests that we have probably only extended the natural history of this indolent disease with repeated relapses at increasingly shorter intervals. Despite recent therapeutic progress and improved OS, FL remains the leading cause of death in patients with a cumulative incidence of $10 \%$ at 10 years. ${ }^{3}$ Nonetheless, even after first relapse/progression the survival data are promising, with a median OS for patients who received second-line treatment beyond 10 years. ${ }^{37}$ Patients experiencing disease progression within 24 months after treatment initiation (POD24) represent a particular group of need, with a 5year survival of only $50 \% .^{38}$ However, it should be noted that this datum was derived in an era that predated PETbased staging and in one large retrospective populationbased study the prognostic impact of POD24 is not as powerful in the modern era. ${ }^{39}$

Histological transformation is probably a turning point for patients' outcome with a shorter survival if it occurs after FL treatment. De novo histological transformation (i.e., diffuse large B-cell lymphoma histology with pathological findings showing existence of a FL component at the time of diagnosis) has a better prognosis. ${ }^{40}$ The median survival after histological transformation is around 4 to 5 years and probably shorter if the transformation occurs within the first year. ${ }^{41}$ The annual incidence of biopsydocumented histological transformation is $<3 \%$, with a plateau after the first 2 years following treatment initiation. ${ }^{11,41}$ One study of more than 8,000 patients identified a lower incidence of histological transformation in the rituximab era. The 10-year cumulative hazard of histological transformation was 5.2\% (95\% CI: 4.5-6.2) in patients who received rituximab and $8.7 \%$ in those who did not (hazard ratio $=0.73,95 \%$ CI: $0.58-0.90 ; \quad P=0.004) .{ }^{42}$ Histological transformation is probably under-reported given that biopsy, while recommended, is not uniformly performed at relapse/progression.

\section{Initial treatment of follicular lymphoma}

After distinguishing localized from disseminated disease applying the Lugano 2014 classification, the GELF/BNLI ${ }^{28,29}$ (Tables 1 and 2) treatment-initiation indices help to identify patients with symptomatic FL requiring treatment. A combination of staging and GELF/BNLI characteristics distinguishes three clinical situations that require different therapeutic approaches: (i) patients with localized FL; (ii) patients with disseminated FL not meeting treatment-initiation criteria (also called "low-tumor burden"); and (iii) patients with disseminated FL who meet treatment-initiation criteria (also called "high-tumor burden").

\section{Localized follicular lymphoma}

Fewer than $20 \%$ of patients present with stage I or II FL at diagnosis. ${ }^{37}$ Such patients are identified after a comprehensive assessment, including both PET and bone marrow biopsy to confirm the localized nature of the disease. Stage I disease, with removal of the only pathological node (stage I0) is an infrequent clinical situation that can benefit from therapeutic abstention. While data are sparse, additional treatment (such as radiotherapy) does not appear to 
Table 4. Survival of patients with follicular lymphoma according to FLIPI, FLIPI-2 and PRIMA-PI.

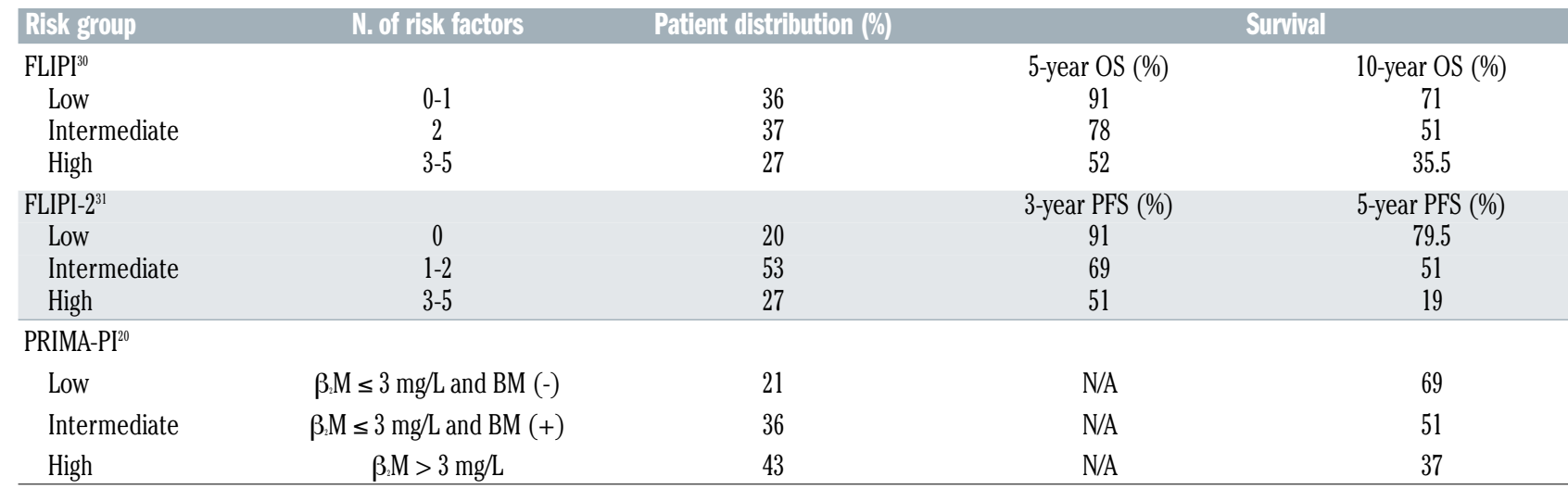

FLIPI: Follicular Lymphoma International :Prognostic Index; PRIMA-PI; PRIMA Prognostic Index; OS: overall survival; PFS: progression-free survival; $\beta$ M: beta 2-microglobulin; BM: bone marrow.

modify the evolution of these patients whose relapse profile is disseminated disease in half of the cases. ${ }^{43}$

For patients with stage I or II FL with persistent lymphadenopathy after diagnostic biopsy, there is no consensus on appropriate therapy. Alternatives to discuss with the patient include abstention ("wait and watch"), radiotherapy, and rituximab and chemotherapy followed by radiotherapy or immunochemotherapy alone. None of these strategies has demonstrated superiority with regard to OS even if immunochemotherapy, ${ }^{44}$ chemotherapy followed by radiation therapy ${ }^{45}$ or radiation followed by chemotherapy ${ }^{46}$ results in improved PFS. If radiotherapy is chosen, a dose of 24 Grays (Gy) in 12 fractions gives similar results to a dose of 40-45 Gy. ${ }^{47}$ Low-dose radiotherapy (4 Gy in 2 fractions) may be preferred for some disease locations to minimize toxicity, particularly when radiotherapy is of palliative intent, but is associated with an inferior PFS. ${ }^{48}$ Thus, for most patients, the physician could recommend either treatment abstention, radiotherapy (24 Gy) or rituximab (4 weekly infusions). ${ }^{44,45}$

Some patients with localized FL have poor prognostic characteristics according to GELF/BNLI criteria or FLIPI and FLIPI-2 scores. For these patients, immunochemotherapy as discussed later for advanced stage symptomatic FL could be considered. These patients include subjects with bulky disease ( $>7 \mathrm{~cm}$ according to GELF, or $6 \mathrm{~cm}$ according to FLIPI-2), particularly abdominal disease, or a lymphomarelated increase of lactate dehydrogenase or $\beta_{2}$-microglobulin levels.

\section{Advanced stage follicular lymphoma without treatment-initiation criteria (low-tumor burden)}

For advanced stage FL without criteria for treatment initiation (asymptomatic disease), the recommendation is usually to propose therapeutic abstention with dynamic observation. There is no demonstrated OS benefit from initiating early treatment. ${ }^{27,28}$ The observation that 15 to $20 \%$ of patients have still not received treatment 10 years after diagnosis, and that $12 \%$ will observe a spontaneous reduction of their disease with an estimated $6 \%$ obtaining a complete response, also argues for a "watch and wait" strategy. These patients must, however, be carefully monitored because the median time to starting treatment is around 31 months. ${ }^{1}$ These clinical observations and the unease that some patients and physicians have with this "watch and wait" strategy led to investigations on the use of rituximab in asymptomatic and disseminated FL. Rituximab monotherapy was examined in two large prospective multicenter trials. ${ }^{1,49}$ In these trials, rituximab was administered in four weekly infusions followed by maintenance rituximab (infusions every 2 months for 2 years or until progression $)^{1}$ or with re-treatment according to the same schedule (4 weekly infusions) at progression. ${ }^{49}$ These two prospective trials demonstrated that after a long follow-up, rituximab monotherapy had no significant side effects. Rituximab increased both PFS and time to the next treatment (TTNT) without having an impact on OS. Ongoing treatment with rituximab maintenance augments the PFS and the TTNT advantage but also the treatment duration (2 years) and increases the rate of grade 1-2 infections.

The USA study also demonstrated that there is no benefit from maintenance treatment compared to re-treatment at progression, with four weekly rituximab infusions, leading to a lower utilization of resources for a similar result.

If "watch and wait" is an appropriate strategy for a given patient with asymptomatic disease, the physician must ensure that the objectives of this strategy are well understood and accepted by the patient or risk undermining the patient's confidence. The patient needs to be aware that this approach does not affect OS and is accompanied by a median time to treatment of 2.5 years. ${ }^{1}$ The physician who proposes this abstention should not underestimate the psychological consequences for the patient and his or her family of the announcement of a malignant disease, not justifying treatment at the moment but being able to justify it in the near future. The delay in initiating treatment is often seen by patients as time lost in the "fight against the disease" and the physician should not underrate the potential negative impact of this strategy. Instead, with psychologist support, the time can be positioned as a period in which to optimize patients' health and address social issues that will affect their fitness and tolerance of therapy, such as weight management, physical fitness and smoking cessation and arranging leave from employment and/or securing extra support from caregivers. Efforts must be made to help the patient understand the proposed lymphoma strategy and to reciprocally understand and acknowledge the patient's experience of their disease. Administration of four weekly injections of rituximab can be an alternative to therapeutic abstention for some patients, for whom compliance with a "watch and wait" proposal appears difficult. The impact of such a strategy on subsequent access to promising first- 
G. Carton and J. Trotman

A

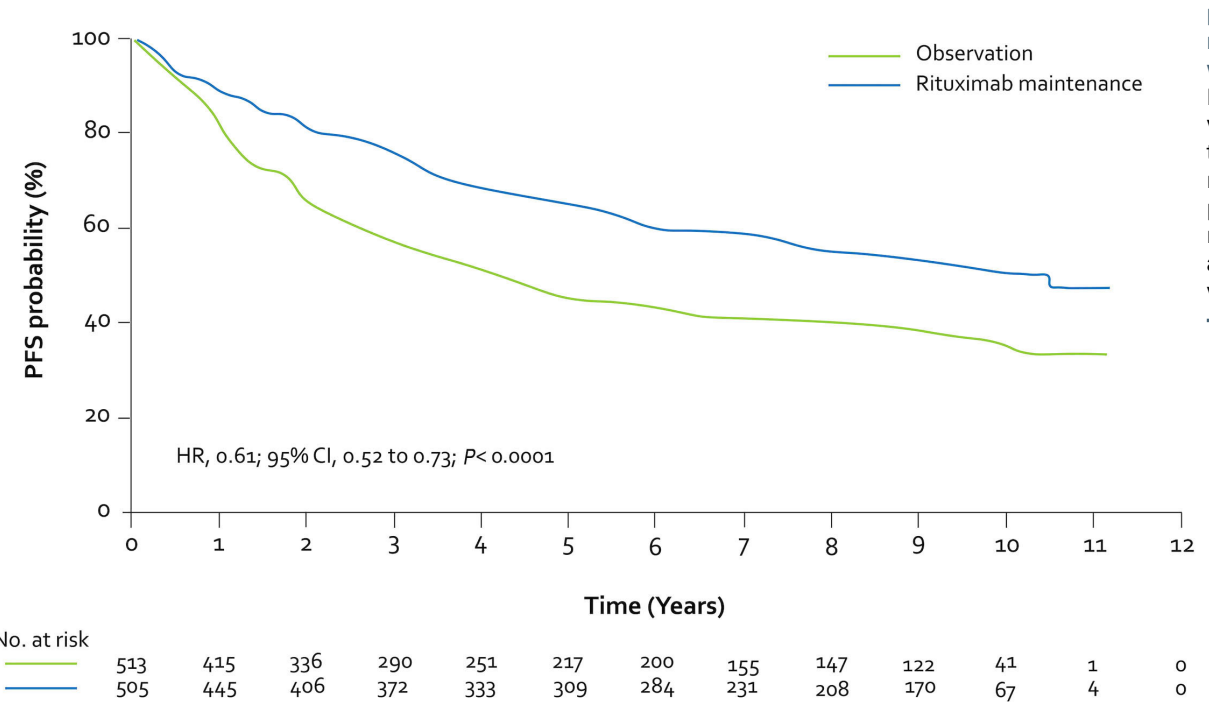

B

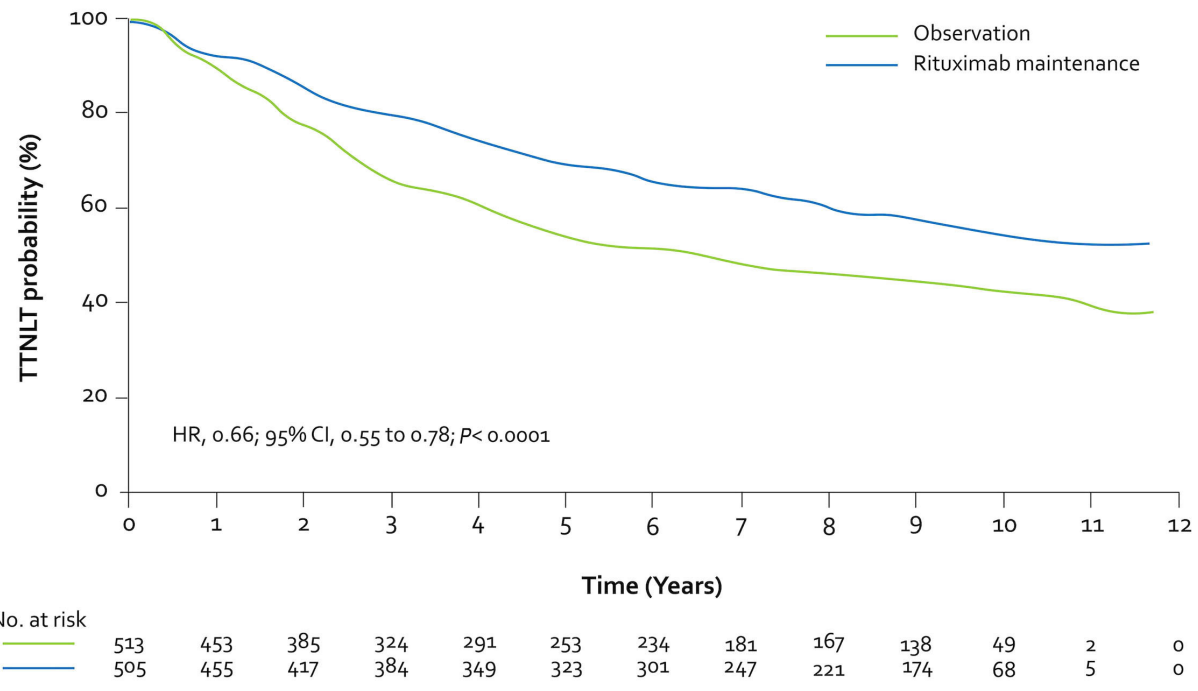

C

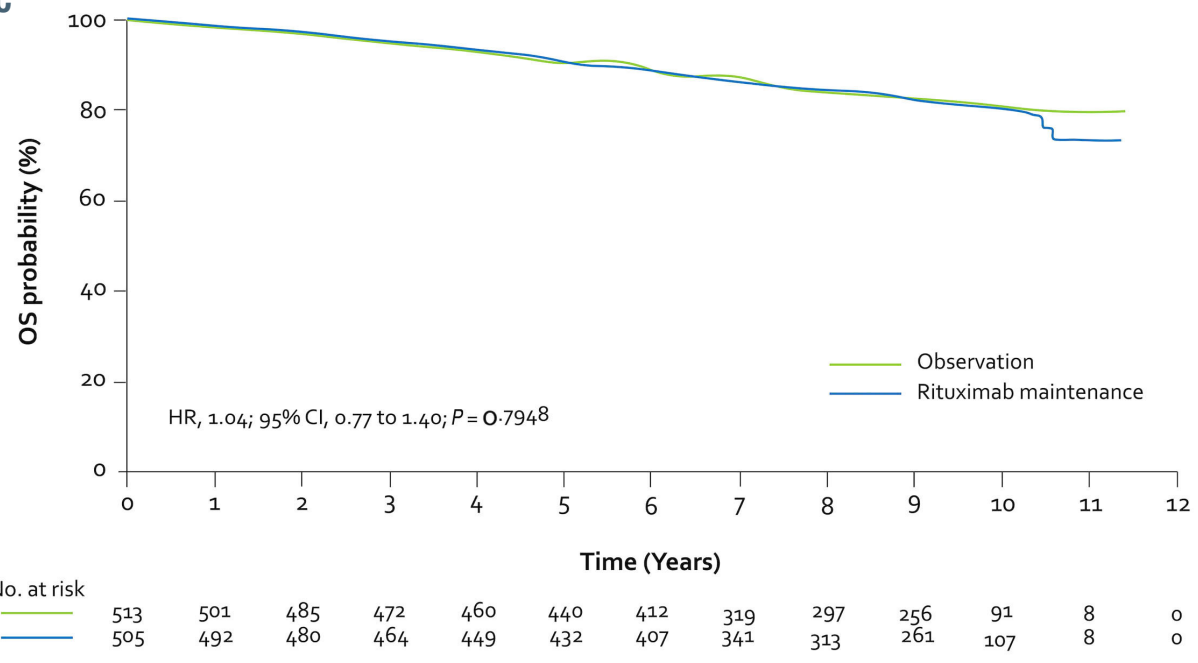

Figure 1. Results of immunochemotheraby with or without rituximab mainenance as first-line treatment in patients with follicular lymphoma. (A-C) KaplanMeier estimates of progression-free surviral (A); time to next anti-lymphoma treatment $(B)$ and overall survival $(C)$ from randomization. OS: overall survival; PFS: progression-free survival; TTNLT: time to next anti-lymphoma treatment; HR: hazard ratio; 95\% Cl: 95\% confidence interval. Adapted from Bachy E, et al. ${ }^{2}$.

12

haematological | 2022; 107(1) 
line treatment options in clinical trials needs to be understood. If maintenance rituximab is provided, a short schema (M3, M5, M7 and M9) is preferred because these give similar results to those of a prolonged schedule. ${ }^{50}$ Because such a recommendation results in complete response in 50 to $70 \%$, without significant side effects, it allows "time without disease and chemotherapy" (option preferred by the patient) instead of "time to wait for treatment" (option preferred by the physician). With rituximab treatment, approximately $25 \%$ of patients will need retreatment within 5 years ${ }^{1}$. In patients for whom "wait and watch" has been chosen, close clinical monitoring (every 3 months) should be carried out during the first year of surveillance, before moving to less intensive observation (every 6 months) in the absence of early signs of progression. Patients must be assured that they will be seen promptly in the event of any concerns about progression. Annual CT scanning may be appropriate to offer and reassure the patient with isolated abdominal disease. The frequency of scanning is ultimately often reduced by those concerned about radiation exposure but for many with stable or only very slowly progressive disease annual imaging provides reassurance that they can continue with work or other life plans without expectation of interruption. The use of PET to monitor FL during the "watch and wait" period is discouraged.

\section{Advanced stage follicular lymphoma with treatment initiation criteria (high-tumor burden)}

\section{Immunochemotherapy improves overall survival}

For disseminated FL that meets criteria for treatment initiation, the benefit of combining rituximab with chemotherapy has been clearly demonstrated in four prospective randomized trials ${ }^{51-54}$ (Table 5.). A Cochrane analysis ${ }^{55}$ including these studies showed a $37 \%$ reduction in the risk of death ( $\mathrm{HR}=0.63,95 \% \mathrm{CI}: 0.51-0.79)$ when rituximab is used in combination with chemotherapy as first-line treatment. Because of the significant decrease of toxicity, rituximab alone has been proposed. This approach cannot, however, be considered as standard treatment for symptomatic patients with FL considering the reduced PFS and TTNT compared to those achieved with immunochemotherapy. ${ }^{56}$

Rituximab maintenance improves progression-free survival but not overall survival

The PFS benefit of maintenance treatment using rituximab was demonstrated in the PRIMA trial. ${ }^{2}$ After induction using immunochemotherapy, with R-CHOP, R-CVP (rituximab plus cyclophosphamide. vincristine, prednisone) or R-FCM (rituximab plus fludarabine, cyclophos- phamide, mitoxantrone), responding patients were randomized to observation or maintenance treatment with rituximab every 2 months for 2 years. With 10 years of follow-up, this trial showed a significant increase in PFS for patients receiving maintenance treatment with a median PFS of 10.5 years versus 4.1 years for those not given maintenance. The median time to the next treatment was not reached in patients receiving maintenance treatment compared to 6.6 years in the control arm. However, this trial did not show an OS benefit for maintenance, with 10-year OS being $80 \%$ in both arms, raising questions about the merits of maintenance, especially in elderly patients or those with respiratory problems in whom toxicity may be less acceptable. The PFS benefit of maintenance treatment with rituximab after immunochemotherapy with bendamustine-rituximab was suggested in an ad hoc analysis in the BRIGHT study. ${ }^{57}$

\section{The best chemotherapy regimen is likely patient-specific}

A number of chemotherapy regimens have been enhanced by their combination with an anti-CD20 antibody (Table 5). One study (Foll05) prospectively compared three different chemotherapy regimens, ${ }^{58} \mathrm{R}-\mathrm{CVP}, \mathrm{R}-\mathrm{CHOP}$ and R-FC (rituximab plus fludarabine and cyclophosphamide), showing that while both R-CHOP and R-FC produced superior PFS and TTNT, in the long-term, the immunosuppressive toxicity of fludarabine precluded it as a first-line therapy. In two other studies R-CHOP was compared with rituximab-bendamustine. ${ }^{59,60}$ Although both studies suffered from methodological issues (nonexclusively FL population, abnormally low results of the RCHOP arm, R-CHOP/R-CVP aggregation) they showed a superior PFS of the rituximab-bendamustine combination. The recent GALLIUM study, ${ }^{61}$ albeit with a non-randomized choice of chemotherapy, was arguably the only study to systematically collect comprehensive toxicity data after bendamustine use. As a counterpoint to an emphasis on PFS as the key consideration when choosing bendamustine as the chemotherapy backbone, the rate of fatal adverse events in GALLIUM was 5.3\% (36/676) in patients assigned to bendamustine as part of induction compared to $1.8 \%(9 / 513)$ in those who received CHOP or CVP after a median follow-up of 41 months. The difference was particularly notable in older patients. The choice of chemotherapy backbone for a given patient is strongly influenced by the patient's age, co-morbidities and treatment preferences (Table 6). Notwithstanding the debate over the potential trade-offs between toxicity and efficacy of CHOP and bendamustine, in patients with a contraindication to anthracyclines, the preference may be for bendamustine in younger patients while the CVP combination may be preferred in elderly patients because of its lower

Table 5. Randomized trials demonstrating the benefit of chemo-immunotherapy on overall survival.

\begin{tabular}{|c|c|c|c|c|c|c|}
\hline \multirow[t]{2}{*}{ Trial } & \multirow[t]{2}{*}{ Treatment } & \multirow[t]{2}{*}{ N. of patients } & \multirow{2}{*}{$\begin{array}{l}\text { Follow-up } \\
\text { (months) }\end{array}$} & \multicolumn{2}{|c|}{ OS $(\%)$} & \multirow[b]{2}{*}{ P } \\
\hline & & & & Control & Rituximab & \\
\hline $\mathrm{M} 3902^{51}$ & $8 \times$ CVP vs $8 \times$ R-CVP & 318 & $53 \mathrm{mo}$ & 77 & 83 & 0.029 \\
\hline $\mathrm{GLSG}^{54}$ & 6-8 x CHOP vs 6-8 x R-CHOP & 428 & $60 \mathrm{mo}$ & 84 & 90 & 0.016 \\
\hline M $39023^{53}$ & 8 x MCP vs 8 x R-MCP & 201 & $47 \mathrm{mo}$ & 74 & 87 & 0.0096 \\
\hline FL2000 52 & $6 \times$ CHVP-I vs $6 \times$ R-CHVP-I & 360 & $100 \mathrm{mo}$ & 70 & 78 & 0.076 \\
\hline
\end{tabular}

OS: overall aurvival; CVP: $750 \mathrm{mg} / \mathrm{m}^{2}$ cyclophosphamide $750 \mathrm{mg} / \mathrm{m}^{2}$ intravenously (iv) day 1 ; vincristine $1.4 \mathrm{mg} / \mathrm{m}^{2}$ (maximum $2 \mathrm{mg}$ ) iv day $1 ; \mathrm{prednisone} 40 \mathrm{mg} / \mathrm{m}^{2}$ orally (po) days 1-5. CHOP: cyclophosphamide $750 \mathrm{mg} / \mathrm{m}^{2}$ iv day 1 ; doxorubicin $50 \mathrm{mg} / \mathrm{m}^{2}$ iv day 1 ; vincristine $1.4 \mathrm{mg} / \mathrm{m}^{2}$ (max, $2.0 \mathrm{mg}$ ) iv day $1 ; \mathrm{prednisone} 100 \mathrm{mg} / \mathrm{m}^{2}$ po days $1-5$. MCP mitoxantrone $8 \mathrm{mg} / \mathrm{m}^{2}$ iv day 1 ; chlorambucil $3 \times 3 \mathrm{mg} / \mathrm{m}^{2}$ po days $1-5$; prednisolone $25 \mathrm{mg} / \mathrm{m}^{2}$ po days $1-5$. CHVP : cyclophosphamide $600 \mathrm{mg} / \mathrm{m}^{2}$ iv day 1 ; doxorubicin $25 \mathrm{mg} / \mathrm{m}^{2}$ iv day 1 ; etoposide $100 \mathrm{mg} / \mathrm{m}^{2}$ iv day 1 ; prednisolone $40 \mathrm{mg} / \mathrm{m}^{2}$ po days $1-5$. I: interferon- $\alpha: 3 \mathrm{x} /$ week for $18 \mathrm{months}, 4.5 \mathrm{MUI}<70$ years ; $3 \mathrm{MUI}>70$ years. R: rituximab: $375 \mathrm{mg} / \mathrm{m}^{2}$ iv 
Table 6. Summary of chemotherapy regimen: advantages and disadvantages.

\begin{tabular}{|c|c|c|}
\hline & Advantages & Disadvantages \\
\hline Bendamustine & $\begin{array}{l}\text { No alopecia } \\
\text { Less febrile neutropenia } \\
\text { No cardiomyopathy or peripheral neuropathy } \\
\text { PFS } \\
\text { TTNT } \\
\text { Lower rate of POD24 }\end{array}$ & $\begin{array}{l}\text { Long-term infectious risk and mortality in patients }>70 \text { years } \\
\text { Early and prolonged CD4 } 4^{+} \text {depletion } \\
\rightarrow \text { Increased risk of severe COVID-19 } \\
\rightarrow \text { Increased risk of lymphocyte harvest failure for CAR T-cell manufacturing }\end{array}$ \\
\hline CHOP & $\begin{array}{l}\text { Effective in } \mathrm{HT} / 3 \mathrm{~B} \text { disease } \\
\text { Lower rates of stem cell toxicity and } \\
\text { persistent immunodeficiency } \\
\text { Lower rates of subsequent HT } \\
\text { Effectiveness in } 3 \mathrm{~A} \text { disease }\end{array}$ & $\begin{array}{l}\text { Increased risk of cardiac toxicity } \\
\text { Peripheral neuropathy } \\
\text { Steroid side effects } \\
\text { Alopecia }\end{array}$ \\
\hline CVP & $\begin{array}{l}\text { Lower infectious toxicity } \\
\text { Less fatigue } \\
\text { Minimal alopecia }\end{array}$ & $\begin{array}{l}\text { Lower PFS/TTNT } \\
\text { Steroid side effects } \\
\text { Peripheral neuropathy }\end{array}$ \\
\hline
\end{tabular}

COVID-19; coronavirus-2019 disease; CAR: chimeric antigen receptor; PFS: progression-free survival; TTNT: time to next treatment; POD24: progression of disease by 24 months HT: histological transformation; CHOP: cyclophosphamide, doxorubicin, vincristine, prednisone; CVP: cyclophosphamide, vincristine, prednisone.

hematologic toxicity. The RELEVANCE study aimed to demonstrate superiority of an immunotherapy combining rituximab and lenalidomide over conventional immunochemotherapy. ${ }^{2}$ The lack of any advantage on both response rates and PFS has dampened hopes of a chemotherapy-free standard-of-care first-line treatment for FL.

\section{Obinutuzumab offers improved progression-free survival but not overall survival}

Obinutuzumab is an anti-CD20 monoclonal antibody modified to improve direct cytotoxicity and antibodydependent cell cytotoxicity. This antibody has been compared to rituximab in a phase III trial (GALLIUM) ${ }^{61}$ combined in induction with different chemotherapy regimens (CHOP, bendamustine, CVP). This immunochemotherapy was followed in responding patients by maintenance treatment with rituximab or obinutuzumab every 2 months for 2 years. It is noted that the dose and administration schedule of the two anti-CD20 antibodies were different, the pharmacokinetic studies carried out during the development of obinutuzumab making it possible to optimize its administration. This study showed that obinutuzumab provides a significant improvement in PFS and TTNT for patients. After a follow-up of 57 months, this translated into risk reductions of $27 \%$ ( $\mathrm{HR}=0.73,95 \% \mathrm{CI}$ : $0.59-0.90)$ for progression and $30 \%(\mathrm{HR}=0.70,95 \% \mathrm{CI}: 0.54,0.90)$ for TTNT. ${ }^{63}$ The advantage of obinutuzumab on PFS and TTNT was especially marked in patients receiving bendamustine. ${ }^{64}$ Nonetheless, mindful that TTNT is arguably a more important endpoint in routine clinical practice than in clinical trials, there was no significant TTNT difference at 3 years across all obinutuzumab-chemotherapy arms: obinutuzumab-bendamustine 87\%, (83-91\%), obinutuzumab-CHOP $87 \%$ (82-91\%), obinutuzumab-CVP $87 \%$ (75-93\%). When using obinutuzumab patients need to be educated about the high probability $(59 \%)$ of an infusionrelated reaction. Reassurance about prompt management of such reactions is important to allay patients' fears and improve their experience with this monoclonal antibody.

The preferred first-line immunochemotherapy approach is patient-specific.

Choosing between combination immunochemotherapy options requires an individualized, nuanced approach for each patient, with risk:benefit calculations made both before induction and during treatment. While there is no direct randomized comparison between induction with bendamustine and CHOP for young fit patients in the modern era, accumulated data suggest that where PFS is the overriding priority this may be best achieved with the use of obinutuzumab-bendamustine followed by continued therapy with obinutuzumab maintenance. Where the anticipated bendamustine toxicity (including that which may impair T-cell function, affecting both immune fitness and potential chimeric antigen receptor T-cell collection) creates a preference for CHOP (particularly where concerns exist regarding occult transformation) or even CVP, their combination with obinutuzumab results in excellent PFS and TTNT with acceptable toxicity. Nonetheless, the GALLIUM study data are early data, and 5 years of follow-up are inadequate for a disease with a median OS beyond 15-20 years. Clinicians are advised to keep this in mind when scheduling first-line therapies for the management of high-tumor burden FL. Mindful of the increased risk of infections related to the use of maintenance with anti-CD20 therapy, ongoing use of such maintenance needs careful reconsideration in the event of any infection and meticulous adherence to antimicrobial prophylaxis is encouraged. Because of increased toxicity related to obinutuzumab (first dose infusion-related reactions, cytopenia and infections), the lack of a demonstrated OS improvement, and cost considerations, rituximab may remain the preferred anti-CD20 antibody, particularly in very frail elderly patients for whom PFS and TTNT are not the overriding clinical objectives. In this setting, the combination of rituximab with CVP offers the lowest toxicity, albeit with a markedly inferior PFS compared to other immunochemotherapy options. Conversely, where chemotherapy toxicity is of concern but PFS remains a priority, pairing less toxic CVP with obinutuzumab with careful management of infusion-related reactions may be a preferred approach for these elderly patients.

\section{Surveillance during first remission in follicular lymphoma}

Current guidelines from the European Society for Medical Oncology advise clinical and imaging surveillance every 6 months for 2 years after induction and, optionally, annually up to 5 years. ${ }^{65}$ Surveillance imaging recommendations were mostly based on data from stud- 
ies of aggressive lymphoma whereas FL has a different disease biology and pattern of relapse. For post-induction PET-negative patients in first remission destined to have a long survival and for whom most relapses will occur after more than 3 years, the cumulative radiation exposure and the increase of life-time incidence of cancer attributable to radiation exposure ${ }^{66}$ do not argue for prolonged imaging surveillance, aside from considerations of increased expense and heightened anxiety. Thus, the limited value of surveillance imaging in detecting asymptomatic nonHodgkin lymphoma relapse earlier, relative to symptomdriven investigations is now generally acknowledged even if not investigated in a randomized controlled manner.

A recent retrospective study analyzing the effect of surveillance imaging on relapse detection and OS concluded that the majority of relapses after first remission are initially suggested by clinical signs and symptoms and relapse detection through routine surveillance imaging in asymptomatic patients carries no additional survival advantage over imaging in the case of clinical concerns. ${ }^{67}$ Although PET-CT is more sensitive than CT-scan for asymptomatic surveillance imaging in $\mathrm{FL}$, it has a lower specificity compared to CT-scan, leading to invasive biopsies and heightening anxiety and cost burden. There is now evidence for recommendations limiting imaging surveillance to examinations that significantly change treatment and/or demonstrate prognostic value. Thus PET-CT at the end of induction and CT scanning every 6 months during the 2 years following induction therapy enable the detection of POD24 patients at risk of early FLrelated death. After that, imaging investigations should be limited to confirmation of relapse in the presence of concerning clinical signs and symptoms. Molecular monitoring for minimal residual disease is not recommended outside of clinical trials.

\section{Ongoing unmet needs in follicular lymphoma}

The estimated prolonged survival of most patients diagnosed with FL in 2021 represents one of the greatest oncology advances in the new millennium. However, this advance is based primarily on the introduction of combined immunochemotherapy induction and maintenance and/or re-treatment with a failure to demonstrate superiority of a lenalidomide-rituximab "chemo-free" approach. While collaborative efforts have studied imaging and molecular markers of response, we still struggle to clearly identify at diagnosis the individual patient destined to have an inferior response for whom therapies used in relapse warrant testing in the first-line setting. With perhaps the future exception of EZH2 inhibitors, a greater understanding of the mutational landscape has not eventuated in the hoped for precision medicine in FL. The biological plausibility of efficacy of BCL-2 and BTK inhibitors has not translated into meaningful clinical benefit and the phophoinositidine-3 kinase inhibitors have remained firmly as therapies for later relapse. Rather, we are currently focused on the potential to harness the power of chimeric antigen receptor $T$ cells and bi-specific antibody therapies in patients with multiply relapsed/refractory disease with hope that success with these cellular therapies will warrant their study in the management of early relapses. Our current focus on the unmet need for the early progressing (POD24) population is appropriate. Patients who have remained event free for 24 months after immunochemotherapy have an age- and gender-matched survival comparable to that of the general population. ${ }^{34}$ However, for such patients destined to have a "functional cure", a clear unmet is the development of appropriate trial designs that move beyond PFS as the sole primary endpoint. Perhaps it is time to develop, in parallel, a composite health utility measure that incorporates both efficacy and toxicity of therapy for such patients with FL.

\section{Impact of the SARS-CoV-2 pandemic on the care of patients with follicular lymphoma}

Coronavirus disease 2019 (COVID-19) has become a global health problem. Despite the arrival of vaccines, SARS-CoV-2 infection disproportionately affects our patients. Those with hematologic malignancies are at higher risk of death from COVID-19. ${ }^{68}$ Indolent lymphoma was an independent predictor of mortality $(\mathrm{HR}=2.19$, 95\% CI: 1.07-4.48) in a retrospective Italian study. ${ }^{69}$ Initial data support expectations that the rate of SARS-CoV-2 IgG seroconversion after vaccination is lower in patients receiving an anti-CD20 monoclonal antibody..$^{70}$ The profound impact of rituximab was recently highlighted in patients with chronic lymphocytic leukemia in whom none of 22 patients exposed to rituximab in the 12 months preceding vaccination responded to the mRNA vaccines. ${ }^{71}$ Circulating rituximab (and obinutuzumab) is found 3 to 6 months after four weekly infusions of the monoclonal antibody and rituximab induces B-cell depletion ${ }^{72}$ which recovers 9 to 12 months after the last infusion. ${ }^{73}$ Treatment with bendamustine, which lowers the CD4+ ${ }^{+}$-lymphocyte count for several months starting as early as after one cycle, ${ }^{64}$ has also recently been suggested as a determinant of severe COVID-1974 and likely also jeopardizes post-vaccination immune responses. General recommendations for FL patients to continue wearing a face mask and maintain social distancing as well as vaccination of all close contacts remain important. For patients with FL not requiring immediate therapy, rituximab could be withheld at least until there is some confidence that the patients exhibit SARS-Cov-2 post-vaccination immunization, and that their contacts and the community as a whole have likewise received vaccination. For symptomatic patients, vaccination could be attempted before treatment initiation for those able to wait at least 2 to 3 months. For patients requiring treatment, the greater immunosuppression, particularly T-cell depletion, associated with bendamustine suggests that this drug should be avoided. The question of maintenance antibody treatment is challenging since induction immunochemotherapy alone likely results in an inability of patients to become immunized for at least 1 year after the last infusion of the antibody. Maintenance therapy prolongs this problem by at least 2 years. In the COVID era the long-term PRIMA follow-up data warrant a closer look. ${ }^{2}$ While the median PFS of patients not receiving rituximab maintenance was only 4.1 years there was a median 9.3 years for time to next chemotherapy in this arm. In future years, booster vaccines, herd immunity and monoclonal antibody therapies for acute COVID-19 may make SAR-CoV-2 infection less of a threat, but vac- 
cine hesitancy and viral evolution globally continue to challenge confidence in achieving a safe community for our patients.

\section{Conclusions}

$\mathrm{FL}$ is an indolent B-cell lymphoma and affected patients now have a very prolonged median OS approaching 20 years. A hallmark of FL is its heterogeneity both at presentation and in the event of relapse. A significant proportion of patients managed with a "watch and wait" approach do not require any therapy, and the use of rituximab monotherapy for treating these patients is likely lower in the current COVID pandemic. With more accurate staging and expansion of therapeutic options in the modern era the treatment of each patient needs to be individualized. Nuanced decisions made in partnership with the patient and their families must account for competing priorities between efficacy and safety as we "play the long game" in lymphoma management. This is particularly important as data make it clear that neither PFS nor POD24 $4^{75}$ are surrogates for OS. The small but cumulative risk of histological transformation mandates biopsy at relapse, especially in patients with early symptomatic progression for whom aggressive approaches may mitigate the poorer prognosis of early histological transformation. Conversely, for other patients a prolonged first remission after either radiotherapy for low-volume, localized disease or chemoimmunotherapy for high-tumor burden may be sustained for several years. In the elderly or those with substantial co-morbidities, FL may not relapse in their lifetime. The lack of an obvious preferred option for first-line chemoimmunotherapy for FL is exacerbated by the challenges and uncertainties of the COVID era. This pandemic brings into sharp focus the importance of attention to not just PFS but also to treatment toxicities and quality of life when choosing initial chemo-immunotherapy and making an individualized risk:benefit analysis of continuing therapy with antibody maintenance.

\section{Disclosures}

GC reports advisory fees from Roche and Celgene as well as speaker's fees and honoraria from Takeda, Roche, Janssen, Celgene, Abbvie, Sanofi, and Gilead. JT reports research funding to her institution from Beigene, Celgene-BMS, Roche, Pharmacyclics, Janssen and Takeda.

\section{Contributions}

The two authors jointly decided the plan and write the paper.

\section{References}

1. Ardeshna KM, Qian W, Smith P, et al. Rituximab versus a watch-and-wait approach in patients with advanced-stage, asymptomatic, non-bulky follicular lymphoma: an open-label randomised phase 3 trial. Lancet Oncol. 2014;15(4):424-435.

2. Bachy E, Seymour JF, Feugier P, et al. Sustained progression-free survival benefit of rituximab maintenance in patients with follicular lymphoma: long-term results of the PRIMA study. J Clin Oncol. 2019;37(31): 2815-2824.

3. Sarkozy C, Maurer MJ, Link BK, et al. Cause of death in follicular lymphoma in the first decade of the rituximab era: a pooled analysis of French and US cohorts. J Clin Oncol. 2019 ; 37(2):144-152.

4. National Cancer Institute Surveillance, Epidemiology, and End Results Program. Cancer stat facts: NHL-follicular lymphoma. Accessed June 11, 2020. https://seer. cancer.gov/statfacts/html/follicular.html

5. Defossez G, Le Guyader-Peyrou S, Uhry Z, et al. Estimations nationales de l'incidence et de la mortalité par cancer en France métropolitaine entre 1990 et 2018 - Volume 1 : Tumeurs solides : Étude à partir des registres des cancers du réseau Francim. https://www.santepubliquefrance.fr/maladies-et-traumatismes/cancers/cancer-dusein/documents/rapport-synthese/estimations-nationales-de-1-incidence-et-de-lamortalite-par-cancer-en-france-metropolitaine-entre-1990-et-2018-volume-1tumeurs-solides-etud

6. Linet MS, Vajdic CM, Morton LM, et al. Medical history, lifestyle, family history, and occupational risk factors for follicular lymphoma: the InterLymph Non-Hodgkin Lymphoma Subtypes Project. J Natl Cancer Inst Monographs. 2014;2014(48):26-40.

7. Schollkopf C, Smedby KE, Hjalgrim H, et al. Cigarette smoking and risk of non-
Hodgkin's lymphoma-a population-based case-control study. Cancer Epidemiol Biomarkers Prev. 2005;14(7):1791-1796.

8. Chiu BC, Dave BJ, Blair A, et al. Agricultural pesticide use and risk of $t(14 ; 18)$-defined subtypes of non-Hodgkin lymphoma. Blood. 2006;108(4):1363-1369.

9. AgopianI, NavarroJM, GacAC, et al. Agricultural pesticide exposure and the molecular connection to lymphomagenesis. J Exp Med. 2009;206(7):1473-1483.

10. Sarkozy C, Baseggio L, Feugier P, et al. Peripheral blood involvement in patients with follicular lymphoma: a rare disease manifestation associated with poor prognosis. Br J Haematol. 2014 ;164(5):659-667.

11. Mir F, Barrington SF, Brown $\mathrm{H}$, et al. Baseline SUVmax did not predict histological transformation in follicular lymphoma in the phase 3 GALLIUM study. Blood. 2020;135 (15):1214-1218

12. Barrington S, Trotman J, Sahin D, et al. Baseline PET-derived metabolic tumor volume metrics did not predict outcomes in follicular lymphoma patients treated with firstline immunochemotherapy and antibody maintenance in the phase III GALLIUM study. Blood. 2018;132(Suppl 1):2882

13. Nath K, Law SC, Sabdia B, et al. Intratumoral $\mathrm{T}$ cells have a differential impact on FDG-PET parameters in follicular lymphoma. Blood Adv. 2021;5(12):26442649

14. Swerdlow SH, Campo E, Pileri SA, et al. The 2016 revision of the World Health Organization classification of lymphoid neoplasms. Blood. 2016;127(20):2375-2390.

15. Trotman J, Fournier M, Lamy $\mathrm{T}$, et al. Positron emission tomography-computed tomography (PET-CT) after induction therapy is highly predictive of patient outcome in follicular lymphoma: analysis of PET-CT in a subset of PRIMA trial participants. J Clin Oncol. 2011;29(23):3194-3200.

16. Tychyj-Pinel C, Ricard F, Fulham M, et al.
PET/CT assessment in follicular lymphoma using standardized criteria: central review in the PRIMA study. Eur J Nucl Med Mol Imaging. 2014;41(3):408-415.

17. Iuminari S, Biasoli I Arcaini I et al. The use of FDG-PET in the initial staging of 142 patients with follicular lymphoma: a retrospective study from the FOLL05 randomized trial of the Fondazione Italiana Linfomi. Ann Oncol. 2013;24(8):2108-2112.

18. Meignan M, Cottereau AS, Versari A, et al. Baseline metabolic tumor volume predicts outcome in high-tumor-burden follicular lymphoma: a pooled analysis of three multicenter studies. J Clin Oncol. 2016;34(30): 3618-3626,

19. Jemaa S, Fredrickson J, Coimbra A, et al. A fully automated measurement of total metabolic tumor burden in diffuse large B-cell lymphoma and follicular lymphoma. Blood. 2019;134(Suppl_1):4666

20. Bachy E, Maurer MJ, Habermann TM, et al A simplified scoring system in de novo follicular lymphoma treated initially with immunochemotherapy. Blood. 2018;132 (1):49-58.

21. Rutherford SC, Herold M, Hiddemann W, et al. Impact of bone marrow biopsy on response assessment in immunochemotherapy-treated lymphoma patients in GALLIUM and GOYA. Blood Adv. 2021;4(8):1589-1593.

22. Dupuis J, Berriolo-Riedinger A, Julian A, et al. Impact of [(18)F]fluorodeoxyglucose positron emission tomography response evaluation in patients with high-tumor burden follicular lymphoma treated with immunochemotherapy: a prospective study from the Groupe d'Etudes des Lymphomes de l'Adulte and GOELAMS. J Clin Oncol. 2012;30(35):4317-4322.

23. Trotman J, Luminari $S$, Boussetta $S$, et al. Prognostic value of PET-CT after first-line therapy in patients with follicular lymphoma: a pooled analysis of central scan review in three multicentre studies. Lancet 
Individualized first-line management of FL

Haematol. 2014 ;1(1):e17-27.

24. Trotman J, Barrington SF, Belada D, et al. Prognostic value of end-of-induction PET response after first-line immunochemotherapy for follicular lymphoma (GALLIUM): secondary analysis of a randomised, phase 3 trial. Lancet Oncol. 2018;19(11):1530-1542.

25. Federico M, Manning D, Versari A, et al. Response oriented maintenance therapy in advanced follicular lymphoma. Results of the interim analysis of the FOLL 12 trial conducted by the Fondazione Italian Linfomi. Hematol Oncol. 2019 ;37(S2):153-154.

26. Pettitt AR, Barrington S, Kalakonda N, et al. NCRI PETReA trial: a phase 3 evaluation of PET-guided, response adapted therapy in patients with previously untreated, advanced stage, high-tumour-burden follicular lymphoma. Hematol Oncol. 2019;37 (S2):67-68.

27. Brice P, Bastion Y, Lepage E, et al. Comparison in low-tumor-burden follicular lymphomas between an initial no-treatment policy, prednimustine, or interferon alfa: a randomized study from the Group d'Etude does Lymphomes Folliculaires. Group d'Etude does Lymphomes de l'Adulte. J Chin Oncol. 1997;15(3):1110-1117.

28. Ardeshna KM, Smith P, Norton A, et al. Long-term effect of a watch and wait policy versus immediate systemic treatment for asymptomatic advanced-stage non-Hodgkin lymphoma: a randomised controlled trial. Lancet. 2003;362(9383):516-522.

29. Bach E, Brice P, Delarue R, et al. Long-term follow-up of patients with newly diagnosed follicular lymphoma in the prerituximab era: effect of response quality on survival-a study from the Group d'Etude les Lymphomes de l'Adulte. J Chin Oncol. 2009 ;28(5):822-829.

30. Solal-Céligny P, Roy P, Colombat P, et al. Follicular lymphoma international prognostic index. Blood. 2004;104(5):1258-1265.

31. Federico M, Belle M, Marsheselli L, et al. Follicular Lymphoma International Prognostic Index 2: a new prognostic index for follicular lymphoma developed by the international follicular lymphoma prognostic factor project. J Olin Oncol. 2009;27(27):4555-4562

32. Pastor A, Jurinovic V, Kridel R, et al. Integration of gene mutations in risk prognostication for patients receiving first-line immunochemotherapy for follicular lemphoma: a retrospective analysis of a prospective clinical trial and validation in a population-based registry. Lancet Oncol. 2015;16(9):1111-1122.

33. Hut S, Meson B, Jas JP, et al. A geneexpression profiling score for prediction of outcome in patients with follicular lymephoma: a retrospective training and validatron analysis in three international cohorts. Lancet Oncol. 2018;19(4):549-561.

34. Maurer MJ, Bachy E, Ghesquières $\mathrm{H}$, et al. 2016 Early event status informs subsequent outcome in newly diagnosed follicular lymephoma. Am J Hematol. 2016;91(11):10961101.

35. Friedberg JW, Taylor MD, Cerhan JR, et al. Follicular lymphoma in the United States: first report of the National LymphoCare Study. J Chin Oncol. 2009;27(8):1202-1208.

36. Barraclough A, Bishton M, Cheah C, et al. The diagnostic and therapeutic challenges of Grade 3B follicular lymphoma. Br J Haematol. 2021;195(1):15-24.

37. Batlevi C, Sha F, Alperovich A, et al. Follicular lymphoma in the modern era: surviral, treatment outcome, and identification of high-risk subgroups. Blood Cancer J. 2020;10(7):74.

38. Casulo C, Byrtek M, Dawson KL, et al. Early relapse of follicular lymphoma after rituximab plus cyclophosphamide, doxorubicin, vincristine, and prednisone defines patients at high risk for death: an analysis from the National LymphoCare Study. J Chin Oncol. 2015;33(23):2516-2522.

39. Batlevi C, Sha F, Alperovich A, et al. Positron-emission tomography-based staging reduces the prognostic impact of early disease progression in patients with follicular lymphoma. Eur J Cancer. 2020;126:78-90.

40. Wagner-Johnston ND, Link BK, Byrtek M, et al. Outcomes of transformed follicular lymphoma in the modern era: a report from the National LymphoCare Study (NLCS). Blood. 2015;126(7):851-857.

41. Sarkozy C, Trneny M, Keri L, et al. Risk factors and outcomes for patients with follicular lymphoma who had histologic transfermotion after response to first-line immunochemotherapy in the PRIMA trial. J Olin Oncol. 2016;34(22):2575-2582.

42. Federico $M$, Callabero Barrigon $M D$, Marsheselli L, et al. Rituximab and the risk of transformation of follicular lymphoma: a retrospective pooled analysis. Lancet Haematol. 2018;5(8):359-367.

43. Soubeyran P, Eghbali H, Trojan M, et al. Is there any place for a wait-and-see policy in stage I0 follicular lymphoma? A study of 43 consecutive patients in a single center. Ann Oncol. 1996;7(7):713.

44. Michallet AS, Libras L, Bauwens D, et al. Early stage follicular lymphoma: what is the clinical impact of the first-line treatment strategy? J Hematol Oncol. 2013;6:45.

45. Friedberg JW, Byrtek M, Link BK, et al. Effectiveness of first-line management strategies for stage I follicular lymphoma: analysis of the National LymphoCare Study. J Olin Oncol. 2012;30(27):3368-3375.

46. MacManus M, Fisher R, Kos D, et al. Randomized trial of systemic therapy after involved-field radiotherapy in patients with early-stage follicular lymphoma: TROG 99.03. J Chin Oncol. 2018;36(29):2918-2925. 47. Lowry L, Smith P, Dian W, et al. Reduced dose radiotherapy for local control in nonHodgkin lymphoma: a randomized phase III trial. Radiother Oncol. 2011;100(1):86-92.

48. Hoskin P, Popova B, Schofield O, et al. 4 Fy versus $24 \mathrm{~Gy}$ radiotherapy for follicular and marginal zone lymphoma (FoRT): long-term follow-up of a multicentre, randomised, phase 3 , non-inferiority trial. Lancet Oncol. 2021;22(3):332-340.

49. Kohl B, Hong F, Williams ME, et al. Rituximab extended schedule or re-treatmont trial for low-tumor burden follicular lymphoma: Eastern Cooperative Oncology Group protocol E4402. J Chin Oncol. 2014;32(28):3096-3102.

50. Ghielmini M, Schmitz SF, Cogliatti SB, et al. Prolonged treatment with rituximab in patients with follicular lymphoma significantly increases event-free survival and response duration compared with the stanhard weekly x 4 schedule. Blood. 2004;103 (12):4416-4423.

51. Marcus R, Imrie K, Solal-Celigny P, et al. Phase III study of R-CVP compared with cyclophosphamide, vincristine, and prednisone alone in patients with previously untreated advanced follicular lymphoma. J Chin Oncol. 2008;26(28):4579-4586.

52. Bach E, Hoot R, Morschhauser F, et al. Long-term follow-up of the FL2000 study comparing CHVP-interferon plus rituximab

in follicular lymphoma. Haematological. 2013;98(7):1107-1114.

53. Herold M, Haas A, Stock S, et al. Rituximab added to first-line mitoxantrone, chlorambucil, and prednisolone chemotherapy followed by interferon maintenance prolongs survival in patients with advanced follicular lymphoma: an East German Study Group Hematology and Oncology Study. J Chin Oncol. 2007;25(15):1986-1992.

54. Hiddemann W, Kneba M, Dreyling M, et al. Frontline therapy with rituximab added to the combination of cyclophosphamide, toxorubicin, vincristine, and prednisone (CHOP) significantly improves the outcome for patients with advanced-stage follicular lymphoma compared with therapy with CHOP alone: results of a prospective randomized study of the German Low-Grade Lymphoma Study Group. Blood. 2005;106 (12):3725-3732

55. Shul H, Bohlius J, Skoetz N, et al. Chemotherapy plus rituximab versus chemotherapy alone for B-cell nonHodgkin's lymphoma. Cochrane Database Syst Rev. 2007;(4):CD003805.

56. Locker S, Østenstad B, Hagberg H, et al. Chemotherapy-free initial treatment of advanced indolent lymphoma has durable effect with low toxicity: results from two Nordic Lymphoma Group trials with more than 10 years of follow-up. J Chin Oncol. 2018;38(33):3315-3323.

57. Kahl B, Burke J, van der Jag R, et al. Assessment of maintenance rituximab after first-line bendamustine-rituximab in patients with follicular lymphoma: an analysis from the BRIGHT trial. Blood. 2017;130(Suppl 1):484.

58. Federico M, Luminary S, Dondi A, et al. RCVP versus R-CHOP versus R-FM for the initial treatment of patients with advancedstage follicular lymphoma: results of the FOLL05 trial conducted by the Fondazione Italian Linfomi. J Chin Oncol. 2013;31(12): 1506-1513

59. Kummel MJ, Niederle N, Maschmeyer G, et al. Bendamustine plus rituximab versus $\mathrm{CHOP}$ plus rituximab as first-line treatment for patients with indolent and mantle-cell lymphomas: an open-label, multicentre, randomised, phase 3. Lancet. 2013;381(9873): 1203-1210.

60. Fin IW, van der Jagt R, Kahl B, et al. Firstline treatment of patients with indolent nonHodgkin lymphoma or mantle-cell lymphoma with bendamustine plus rituximab versus $\mathrm{R}-\mathrm{CHOP}$ or R-CVP: results of the BRIGHT 5-year follow-up study. J Chin Oncol. 2019;37(12):984-991.

61. Marcus R, Davies A, Ando K, et al. Obinutuzumab for the first-line treatment of follicular lymphoma. N Angl J Med. 2017; 377(14):1331-1344

62. Morschhauser F, Fowler N, Bouabdallah R, et al. Rituximab plus lenalidomide in advanced untreated follicular lymphoma. N Engl J Med. 2018;379(10):934-947.

63. Townsend W, Burke C, Carton G, et al. Obinutuzumab-based immunochemotheraby prolongs progression-free survival and time to next anti-lymphoma treatment in patients with previously untreated follicular lymphoma: four-year results from the phase III GALLIUM study. Blood. 2018;132(Suppl 1):1597.

64. Hiddemann W, Barbui AM, Canals MA, et al. Immunochemotherapy with obinutuzumab or rituximab for previously untreated follicular lymphoma in the GALLIUM study: influence of chemotherapy on 
efficacy and safety. J Clin Oncol. 2018;36 (23):2395-2404

65. Dreyling $M$, Ghielmini M, Rule S, et al. Newly diagnosed and relapsed follicular lymphoma: ESMO clinical practice guidelines for diagnosis, treatment and follow-up. Ann Oncol. 2020;32(3):298-308.

66. Shenoy P, Sinha R, Tumeh JW, et al. Surveillance computed tomography scans for patients with lymphoma: is the risk worth the benefits? Clin Lymphoma Myeloma Leuk. 2010;10(4):270-277.

67. Goldman MX, Mao JJ, Strouse CS, et al. Surveillance imaging during first remission in follicular lymphoma does not impact overall survival. Cancer. 2021:127(18):33903402.

68. Bhaskaran K, Bacon S, Evans SJ, et al. Factors associated with deaths due to COVID-19 versus other causes: population-based cohort analysis of UK primary care data and linked national death registrations within the OpenSAFELY platform. Lancet Reg Health Eur. 2021;6:100109.

69. Passamonti F, Cattaneo C, Arcaini L, et al. Clinical characteristics and risk factors associated with COVID-19 severity in patients with haematological malignancies in Italy: a retrospective, multicentre, cohort study. Lancet Haematol. 2020;7(10):e737-747.

70. Thakkar A, Pradhan K, Shawn Jindal S, et al Patterns of seroconversion for SARS-CoV-2 IgG in patients with malignant disease and association with anticancer therapy. Nature Cancer. 2021;2:392-399.

71. Herishanu Y, Avivi I, Aharon A, et al. Efficacy of the BNT162b2 mRNA COVID-19 vaccine in patients with chronic lymphocytic leukemia. Blood.2021;137(23):3165-3173.

72. Cartron G, Blasco H, Paintaud G, et al Pharmacokinetics of rituximab and its clinical use: thought for the best use? Crit Rev
Oncol Hematol. 2007:62(1):43-52.

73. Dunleavy K, Hakim F, Kim Hk, et al. B-cell recovery following rituximab-based therapy is associated with perturbations in stromal derived factor- 1 and granulocyte homeostasis. Blood. 2005;106(3):795-802.

74. Lamure S, Dulery R, Di Blasi R, et al Determinants of outcome in Covid-19 hospitalized patients with lymphoma: a retrospective multicentric cohort study. EclinicalMedicine. 2020;27:100549.

75. Bachy E, Cerhan JR, Salles G. Early progression of disease in follicular lymphoma is a robust correlate but not a surrogate for overall survival. Blood Adv. 2021;5(6):1729-1732.

76. Cheson BD, Fisher RI, Barrington SF, et al. Recommendations for initial evaluation, staging, and response assessment of Hodgkin and non-Hodgkin lymphoma: the Lugano classification. J Clin Oncol. 2014;32 (27):3059-3067 